\title{
Auditory Processing in Children with Specific Language Impairments: Are there Deficits in Frequency Discrimination, Temporal Auditory Processing or General Auditory Processing?
}

\author{
Andreas Nickisch Claudia Massinger \\ Department of Phoniatrics and Pedaudiology, Kinderzentrum München gGmbH, and Institute of Social Pediatrics, \\ Ludwig Maximilian University Munich, Munich, Germany
}

\section{Key Words}

Nonverbal auditory processing $\cdot$ Specific language impairment $\cdot$ Frequency discrimination $\cdot$ Intensity discrimination - Gap detection - Time order judgment • Just noticeable differences

\begin{abstract}
Background/Aims: Specific language impairment (SLI) is believed to be associated with nonverbal auditory (NVA) deficits. It remains unclear, however, whether children with SLI show deficits in auditory time processing, time processing in general, frequency discrimination (FD), or NVA processing in general. Patients and Methods: Twenty-seven children (aged 8-11) with SLI and 27 control children (CG), matched for age and gender, were retrospectively compared with regard to their performance on five NVA skills in terms of just noticeable differences (JND) and time order judgments (TOJ). JND was used for FD, intensity discrimination, and gap detection, while TOJ was used for FD and clicks. $\boldsymbol{R e}$ sults: Children with SLI performed significantly worse than the CG only on the FD tasks (JND and TOJ). The other nonverbal tasks showed no significant intergroup differences. Additionally, moderate associations were found between the FD tasks and phonological skills, as well as between FD tasks
\end{abstract}

and language scores. Conclusion: Children with SLI appear to have restricted FD skills compared to controls, but there was no evidence for a common NVA deficit or reduced temporal auditory abilities.

Copyright $\odot 2009$ S. Karger AG, Basel

Specific language impairment (SLI) describes a condition of markedly delayed language acquisition with normal nonverbal cognitive skills. Although peripheral hearing is unaffected, reduced nonverbal auditory (NVA) processing has often been described in children with SLI. Such a diagnosis may include specific impairments in auditory temporal processing, general deficits in temporal processing, or problems in specific auditory functions such as frequency discrimination (FD).

A deficit in NVA temporal processing may reduce the efficiency of discrimination and identification of spectrally complex sounds that change rapidly over time. Therefore, the perception of speech sounds may be distorted, resulting in unstable and reduced neural representations of phonemes during language development, which interfere with both the comprehension and the production of speech [1]. This hypothesis was supported by certain studies using the Rapid Auditory Test [e.g., 2,

\section{KARGER}

Fax +41613061234 E-Mail karger@karger.ch www.karger.com
(C) 2009 S. Karger AG, Basel

$1021-7762 / 09 / 0616-0323 \$ 26.00 / 0$

Accessible online at:

www.karger.com/fp
Dr. Andreas Nickisch

Abteilung für Phoniatrie und Pädaudiologie, Kliniken des Bezirks Oberbayern

Kommunalunternehmen, Kinderzentrum München, Heiglhofstrasse 63

DE-81377 Munich (Germany), Tel. +49 8971009 0, Fax +49 8971009277

E-Mail andreas.nickisch@lrz.uni-muenchen.de 
3], but other authors have since failed to replicate these findings [4-8]. Associations between the Rapid Auditory Test and reading abilities have been identified [9], but none have been shown to exist for language skills $[10,11]$. The general temporal processing deficit hypothesis contends that deficits in auditory temporal processing indicate a more general impairment in processing rapidly changing transient information [11]. This theory is compatible with the generalized slowing hypothesis [e.g., 12], which presumes that children with SLI respond more slowly than normally developing controls (NDCs) in linguistic as well as nonlinguistic tasks [12]. Although children with SLI scored poorly on speed-related tasks compared to NDCs, no correlations between test scores and severity of language impairment were observed [13]. In contrast to the aforementioned hypothesis, people with SLI exhibited problems in specific auditory functions, showing poorer abilities in the following tasks as compared to NDCs: FD of tones in behavioral tests and ERPs [14-16], intensity discrimination of tones in behavioral tests [17], differentiation between two different and fixedfrequency tones without using a time-processing paradigm in both behavioral tests [18] and MMN [e.g., 19], and FD for long as well as short interstimulus intervals in children with SLI in behavioral tests $[4,6]$. Furthermore, researchers have identified significant moderate to strong correlations between FD scores and phonological/speech skills [15]. Reduced abilities in FD may affect discrimination and identification of spectral patterns of speech sounds, and these abilities influence the development of neural representations for the different phonemes during language development [5].

The contradictory findings of NVA processing tasks in children with SLI do not clearly support or undermine any one of the described hypotheses, and further, this contradiction might be explained by:

- insufficient study population size, with no more than

17 participants in any SLI group [2, 6-8, 14-17, 20] while only a few study populations included 25 participants or more $[3,4]$;

- too broad an age range, between 10 or 12 and 20 years [14-16];

- examination of only one or two different aspects of NVA abilities $[4,6,14,18]$. Only a few authors have investigated three or more different aspects in children with SLI as compared to NDC subjects. Two studies $[10,20]$ featured SLI groups $(\mathrm{n}=10$ and 11$)$ that were not large enough to generalize the results. Corriveau et al. [21] found poorer scores in 21 children with SLI compared to NDC subjects in discrimination of amplitude envelope rise time, detecting rise time of two ramps in a tone, and differentiating tone durations, but not in tests that involved tone intensity or a time order judgment (TOJ) task with two different sounds.

Furthermore, to date, there have been no comprehensive reports of behavioral NVA findings in Germanspeaking children with SLI that assess the described findings in languages other than English.

Therefore, the purpose of our study was to compare systematically different NVA abilities (FD, gap detection, tone intensity) with different task qualities (TOJ and just noticeable differences, JND) in a larger group of German-speaking children (aged 8-11) with SLI and age- and gender-matched NDCs. If children with SLI have specific NVA deficits in FD, we would expect differences between children with SLI and the NDC only in the FD-related tasks. Associations between these tests and phonological skills as well as language abilities would be anticipated, indicating their importance for language development.

\section{Participants}

All children in the SLI group were assessed in our Phoniatrics-Pedaudiology Department between 2003 and 2006. All children aged $8 ; 0-11 ; 0$ years diagnosed with SLI and with fully documented medical records were retrospectively selected from our database. The NDCs were recruited from an elementary school (grades 3 and 4 ) and had no history of speech disorders, dyslexia, or hearing impairments. The control participants were matched for age and gender to the children with SLI. Both the SLI group and the control group (CG) consisted of 16 boys and 11 girls.

All children (SLI and CG) demonstrated normal results in tympanometry and pure-tone audiometry (thresholds of $15 \mathrm{~dB}$ and better between 500 and 8,000 $\mathrm{Hz}$ ). All children with SLI had a performance or nonverbal IQ (Hamburg Wechsler Intelligence Scale for Children-III or Kaufman-ABC) of at least 85 (mean 97.3, SD 7.6; normally distributed as confirmed by the Kolmogorov-Smirnov Test). The children did not show any neurological abnormalities, oral structure problems, or autistic disorders in ENT, neuropediatric, and psychological examinations.

All children (SLI and CG) underwent standardized testing of language skills using two subtests of the 'Heidelberger Sprachentwicklungstest' (Heidelberger Speech 
Table 1. Comparison of children with SLI to control children with regard to age, and the results of testing expressive and receptive language skills (raw scores of the CGS and IGS subtests of the HSDT)

\begin{tabular}{lcclllc}
\hline \multirow{2}{*}{ Measure } & \multicolumn{2}{l}{ Means $(95 \% \mathrm{CI})$} & & \multicolumn{2}{c}{ Comparison ( $\mathrm{t}$ test) } \\
\cline { 2 - 3 } & SLI group $(\mathrm{n}=27)$ & CG $(\mathrm{n}=27)$ & & & $\mathrm{t}(52)$ & $\mathrm{p}$ \\
\hline Age, years & $9.6(9.4-9.9)$ & $9.7(9.3-9.9)$ & & & 0.085 & 0.69 \\
IGS & $13.6(11.4-15.8)$ & $23.1(22.4-23.8)$ & & 8.30 & $<0.001$ \\
CGS & $12.2(11.2-13.2)$ & $15.8(15.4-16.2)$ & & 7.01 & $<0.001$ \\
\hline
\end{tabular}

Means are shown together with $95 \%$ confidence intervals (CI) in parentheses as well as $t$ and $p$ values for $t$ tests.
Development Test, HSDT) [22]. The HSDT determines the state of development of linguistic abilities in Germanspeaking children and consists of 13 subtests. The following two subtests (Guttmann's lambda between 0.80 and 0.86 [22]) were applied: the Comprehension of Grammatical Structure Forms (CGS), which aims to examine the ability to understand subject-object differentiations in verbal instructions by acting them out, and the Imitation of Grammatical Structure Forms (IGS), which assesses the accurate repetition of longer sentences.

All tests were administered by experienced language therapists. For all children in the SLI group, the diagnosis of SLI was made during a spontaneous dialogue and was additionally confirmed if at least two of five selected HSDT subtests yielded values more than 1.0 SD below the mean of the reference population. Our standard clinical diagnostic procedure for children with SLI involves five subtests: IGS, CGS, and another three HSDT subtests (Correction of Semantically Inconsistent Sentences, Word Finding, and Sentence Construction).

One-way ANOVA (table 1) did not show any group differences in age $[\mathrm{T}(52)=0.085, \mathrm{p}=0.69]$. As expected, there were between-group differences for both language scores [expressive: $\mathrm{T}(52)=8.30, \mathrm{p}<0.001$; receptive: $\mathrm{T}(52)=7.01, \mathrm{p}<0.001]$, with children in the SLI group exhibiting markedly lower mean expressive and receptive quotients (table 1).

\section{Outcome Measures}

NVA abilities were examined using the PsychoAcoustical TestSYstem (PATSY; Pilot, Blankenfelde, Germany), which contains the following five subtests: JND for Tone Intensity (I-JND), JND for Tone Frequency (F-JND), JND for Gaps in Noise (G-JND), Monaural TOJ with two fixed-frequency tones and varying interstimulus intervals (F-TOJ), and Binaural TOJ (B-TOJ) with the present- ed clicks changing between the ears and varying interstimulus intervals (ISI).

All subtests were performed as a two-alternative forced-choice procedure ( 2 up, 1 down with adaptive step width, automatic system algorithms, and visual feedback). Termination criteria included changing direction at least twice within the minimum step width, test value at the maximum or minimum value, or reaching a maximum of 60 trials. The parameters for F-JND were as follows: initial stimulus $1,000-\mathrm{Hz}$ sine tone, initial difference $100 \mathrm{~Hz}$, range of difference $0.5-260 \mathrm{~Hz}$, step width 10,5 , or $1 \mathrm{~Hz}$, sound duration $300 \mathrm{~ms}$ including onset and offset time intervals of $20 \mathrm{~ms}$ each at $75 \mathrm{~dB}$, and ISI 600 $\mathrm{ms}$. The parameters for I-JND were as follows: initial stimulus $65 \mathrm{~dB}$, initial difference $5 \mathrm{~dB}$, range of difference $0.25-12 \mathrm{~dB}$, step width 2,1 , or $0.25 \mathrm{~dB}, 1,000-\mathrm{Hz}$ sine tone, sound duration $300 \mathrm{~ms}$ including onset and offset time intervals of $20 \mathrm{~ms}$ each, and ISI $600 \mathrm{~ms}$. The parameters for G-JND were as follows: initial gap $40 \mathrm{~ms}$, range of difference 1-85 ms, step width 10,5 , or $2 \mathrm{~ms}, 1,000 \mathrm{~Hz}$ bandpass noise, sound duration $300 \mathrm{~ms}$ including onset and offset time intervals of 1-50 ms at $75 \mathrm{~dB}$, and ISI 600 $\mathrm{ms}$. The parameters for F-TOJ were as follows: initial ISI $400 \mathrm{~ms}$, range of ISI $1-1,145 \mathrm{~ms}$, step widths 100,25 , or $1 \mathrm{~ms}, 1.0$ - and $1.92-\mathrm{Hz}$ sine tones, sound duration $10 \mathrm{~ms}$ including onset and offset time intervals of $1 \mathrm{~ms}$ at $75 \mathrm{~dB}$. The parameters for B-TOJ were as follows: initial ISI 300 $\mathrm{ms}$, range of difference $1-1,000 \mathrm{~ms}$, step widths 50,10 , or $1 \mathrm{~ms}$, click duration $10 \mathrm{~ms}$ including onset and offset time intervals of $1 \mathrm{~ms}$ at $75 \mathrm{~dB}$. The mean retest reliability of PATSY was $r=0.66$ [23]. We used the results (raw scores) of the first test trial for data analysis.

To investigate phonological skills, we applied the Heidelberger Lautdifferenzierungstest (Heidelberger Test of Phoneme Differentiation, HTPD [24]), a widely used test for German-speaking elementary school children (Cronbach's alpha $=0.89-0.95$ [21]). Two of the three subtests contained 25 word pairs that included real words and 
Table 2. Comparison of children with SLI to the control children for NVA skills: I-JND, F-JND, and G-JND, F-TOJ and B-TOJ

\begin{tabular}{lllllll}
\hline \multirow{2}{*}{ Measure } & \multicolumn{2}{l}{ Median, IR, $\mathrm{n}>\mathrm{IR}$} & & \multicolumn{2}{c}{ Comparison (Mann-Whitney U test) } \\
\cline { 2 - 3 } & SLI group $(\mathrm{n}=27)$ & $\mathrm{CG}(\mathrm{n}=27)$ & & & $\mathrm{p}$ (two-tailed) & $\mathrm{r}$ (effect size) \\
\hline I-JND & $3 \mathrm{~dB}(2-6 \mathrm{~dB}) ; 8 / 27$ & $4 \mathrm{~dB}(2-5 \mathrm{~dB}) ; 6 / 27$ & & 347.0 & 0.76 & -0.04 \\
F-JND & $126 \mathrm{~Hz}(85-155 \mathrm{~Hz}) ; 21 / 27$ & $24 \mathrm{~Hz}(7-79 \mathrm{~Hz}) ; 6 / 27$ & & 133.5 & $<0.001$ & -0.54 \\
G-JND & $5 \mathrm{~ms}(3-36 \mathrm{~ms}) ; 10 / 27$ & $5 \mathrm{~ms}(3-8 \mathrm{~ms}) ; 6 / 27$ & & 355.5 & 0.88 & -0.02 \\
F-TOJ & $400 \mathrm{~ms}(69-731 \mathrm{~ms}) ; 19 / 27$ & $26 \mathrm{~ms}(24-101 \mathrm{~ms}) ; 6 / 27$ & & 146.5 & $<0.001$ & -0.50 \\
B-TOJ & $91 \mathrm{~ms}(51-281 \mathrm{~ms}) ; 10 / 27$ & $78 \mathrm{~ms}(30-112 \mathrm{~ms}) ; 6 / 27$ & & 264.0 & 0.18 & -0.18 \\
\hline
\end{tabular}

Medians are given for raw scores, with interquartile ranges (IR) in parentheses as well as $U$ and $p$ values for Mann-Whitney U tests, and the number of individuals showing test results poorer than the IR of the control group $(\mathrm{n}>\mathrm{IR})$.

Table 3. Spearman's rho correlation coefficients between the nonverbal auditory tests and 'language scores' as well as 'phonological scores' for the entire study population (SLI children and controls)

\begin{tabular}{|c|c|c|c|c|}
\hline \multirow[t]{3}{*}{ Measure } & \multicolumn{4}{|c|}{ Raw scores $(\mathrm{n}=54$ children $)$} \\
\hline & \multicolumn{2}{|c|}{ phonological score } & \multicolumn{2}{|c|}{ language score } \\
\hline & rho & $\mathrm{p}$ & rho & $\mathrm{p}$ \\
\hline I-JND & -0.172 & 0.213 & -0.065 & 0.640 \\
\hline F-JND & -0.393 & $0.003^{*}$ & -0.544 & $<0.001^{*}$ \\
\hline G-JND & -0.077 & 0.581 & -0.198 & 0.151 \\
\hline F-TOJ & -0.556 & $<0.001^{*}$ & -0.525 & $<0.001^{*}$ \\
\hline B-TOJ & -0.257 & 0.066 & -0.171 & 0.225 \\
\hline
\end{tabular}

Nonverbal auditory tests: I-JND, F-JND, and G-JND, F-TOJ and B-TOJ. Significance after Bonferroni's correction: ${ }^{*} \mathrm{p}<0.005$ (uncorrected $\alpha$ threshold was 0.05).

nonsense words. The word pairs were either comprised of two identical words (e.g., Dreck - Dreck) or a minimal pair (e.g., Drachen - krachen); the task was to verbally indicate whether the word pair was 'equal' or 'different' (phoneme differentiation subtest) and repeat the word pair (phoneme identification subtest). In the third subtest (phoneme analysis), the children had to analyze and specify the first two phonemes of 12 single words with a consonant-consonant-vowel combination at the beginning of each word.

The raw scores from the three HTPD subtests were combined to give a total 'phonological score', and the raw scores of the two HSDT subtests were summed to give a net 'language score'.
Descriptive statistics were generated using the software package SPSS for Windows 15.0. For three of the five NVA tests and the language score, the KolmogorovSmirnov test resulted in $p$ values $<0.1$ (G-JND: $p<0.001$, F-TOJ: $\mathrm{p}=0.004, \mathrm{~B}-\mathrm{TOJ}: \mathrm{p}=0.001$, and language score $\mathrm{p}=0.024$ ), suggesting that the data were not normally distributed, whereas a normal distribution could be assumed for I-JND ( $\mathrm{p}=0.620)$, F-JND ( $\mathrm{p}=0.135)$, and the phonological score $(\mathrm{p}=0.497)$. Therefore, all group comparisons were analyzed using the Mann-Whitney U test (two-tailed). Spearman's correlations were analyzed between the five NVA variables and the net phonological and language scores. Following the recommendation to report individual scores rather than just group means [25], we calculated how many individuals showed results below the interquartile range of the CG in the NVA tasks and performed the $\chi^{2}$-test.

\section{Results}

Descriptive statistics for the five NVA tasks of SLI children and the CG are listed in table 2: significant, large group differences were found regarding F-JND (24 vs. 126 $\mathrm{Hz}$, respectively) and F-TOJ (26 vs. $400 \mathrm{~ms}$, respectively). In contrast, no differences were seen regarding I-JND, GJND, and B-TOJ. Furthermore, the number of individuals with test results below the interquartile range of the CG for both children with SLI and the CG is listed in table 2. A $\chi^{2}$-test confirmed group differences only for F-JND $\left(\chi^{2}=16.67 ; \mathrm{p}<0.001\right)$ and F-TOJ $\left(\chi^{2}=13.75 ; \mathrm{p}<0.001\right)$, but not for the other NVA tasks (G-JND: $\chi^{2}=1.42 ; \mathrm{p}=0.23$; I-JND: $\chi^{2}=0.39 ; \mathrm{p}=0.54 ; \mathrm{B}-\mathrm{TOJ}: \chi^{2}=1.92 ; \mathrm{p}=0.17$ ). 
Spearman's correlation coefficients were computed to examine associations between the NVA tests and both phonological abilities and language skills (table 3). Significant (moderate) associations were found only in FJND and F-TOJ, both for phonological skills and the language score. After Bonferroni's correction, all recorded correlations remained significant. The remaining NVA tests did not show any significant associations with phonological or language skills. Furthermore, no correlations were identified between the five NVA scores and nonverbal IQ ( $\mathrm{p}>0.23$ for all). Only F-JND exhibited strong correlations with F-TOJ $(\mathrm{r}=0.65, \mathrm{p}<0.001)$, although the other NVA tasks did not exhibit any significant correlations with each other.

\section{Discussion}

Our results clearly show that children with SLI may not be expected to have general deficits in NVA abilities because only two of the five NVA tasks showed significant group differences, with poorer scores for children with SLI. Furthermore, for SLI children, there was no evidence of restrictions in temporal auditory processing, as suggested Tallal [2] and Tallal et al. [3], because the children with SLI in our tests showed significant deficits only in the F-TOJ task and not in the other two tests of time-processing abilities (G-JND and B-TOJ). In fact, children with SLI achieved poorer results only in the two FD tasks (JND and TOJ), indicating the presence of specific deficits in FD. These results are consistent with previous findings of FD deficits in people with SLI $[5,15,16,26]$. In contrast to these studies, some authors observed restricted FD abilities only under time processing demands in children with SLI [e.g., 2], but not in FD tasks using fixed-frequency tones without time processing demands. However, a slight FD deficit may not influence the results of FD tasks with fixed-frequency tones (e.g., 100 vs. $305 \mathrm{~Hz}$ [2]), but it could become more apparent by adding a time-processing task or by determining the threshold frequency difference as in our FD tasks for both conditions. Taking these aspects into account, findings with fixed-frequency tones [e.g., 2, 4] are not necessarily contradictory to our results. Furthermore, Tallal's [2] findings might not necessarily have been caused by time processing problems in children with SLI, but could have been induced by slight FD deficits in children with SLI. Poor performance in FD may reflect spectral rather than temporal processing of auditory signals, particularly speech sig- nals [16]. Impaired spectral processing abilities may be associated with problems in distinguishing similarsounding phonemes that differ primarily in terms of frequency transitions, regardless of their intensity or presentation rate. Instabilities of phoneme discrimination and phoneme identification might interfere with encoding and producing speech, which would ultimately lead to expressive and receptive language disorders [5]. In our study, we were able to replicate constraints of FD in German-speaking children with SLI. Therefore, we confirmed that lower FD scores in children with SLI are detectable in a language other than English.

The strength of our data lies in the examination of not only one or two NVA tasks in 8- to 11-year-old children with SLI and their age-matched controls, but rather five different NVA tests, including JND tasks (FD, intensity discrimination, gap detection) and TOJ tasks (fixed-frequency tones or clicks). Applying these five different NVA test modalities results in a more substantial overview of NVA skills in children with SLI compared to previous studies.

There are, however, some limitations to our study that must be taken into account when interpreting our data. Although our group size is relatively large compared to other studies, even a group size of 27 is unlikely to include the full distribution of the various SLI subtypes, which results in a limited generalizability of the reported findings and their interpretations. Furthermore, although we examined nonverbal intellectual skills in the SLI group, we did not test this in the CG. However, our CG showed normally distributed standard scores of digit span (Kolmogorov-Smirnov's $\mathrm{p}=0.45$ ) that were within the normal range $($ mean $=48.2, \mathrm{SD}=10.2$ ). Additionally, all children with SLI showed nonverbal IQ scores of 85 or better with normally distributed IQ values (KomogorovSmirnov's $p=0.782$ ) and a mean IQ of 97.3, which we believe is similar to that of the general population. Furthermore, for the SLI group, we detected no associations between the IQ scores and any NVA task; thus, we do not believe that there were any fundamental effects of nonverbal IQ on our auditory processing results. Although the differences in FD that were identified between children with SLI and the CG appear to provide an important contribution to our understanding of deficits in children with SLI, only a fraction of our children with SLI showed poor FD abilities, with only moderate associations between these tasks and language skills. Therefore, these NVA abilities cannot be assumed to be factors that uniquely cause SLI. 
Further research must thus be conducted to assess the incidence of reduced FD in children with normal language development. Children with SLI and normal FD skills should be differentially examined in their language skills to determine differences in language profiles between these children with SLI and those with restricted FD skills. This research would clarify the importance of FD measures in working with SLI patients.

\section{References}

1 Tallal P: Experimental studies of language learning impairments: from research to remediation; in Bishop DVM, Leonard LB (eds): Speech and Language Impairments in Children. Hove, Psychology Press, 2000, pp. 131-155.

2 Tallal P: Rapid auditory processing in normal and disordered language development. Speech Hear Res 1976;19:561-571.

$\checkmark 3$ Tallal P, Stark RE, Kallman C, Mellits D: A re-examination of some nonverbal perceptual abilities of language-impaired and normal children as a function of age and sensory modality. J Speech Hear Res 1981;24:351357.

4 Bishop DVM, Bishop SJ, Bright P, James C, Delaney T, Tallal P: Different origin of auditory and phonological problems in children with language impairment: evidence from a twin study. J Speech Lang Hear Res 1999;42: 155-168.

5 McArthur GM, Bishop DVM: Frequency discrimination deficits in people with specific language impairment: reliability, validity, and linguistic correlates. J Speech Lang Hear Res 2004;47:527-541.

6 Heath SM, Hogben JH, Clark CD: Auditory temporal processing in disabled readers with and without oral language delay. J Child Psychol Psychiatry 1999;40:637-647.

$\checkmark 7$ Helzer JR, Champlin CA, Gillam RB: Auditory temporal resolution in specifically language-impaired and age-matched children. Percept Mot Skills 1996;83:1171-1181.

$\checkmark 8$ Fernell E, Norrelgen F, Bozkurt I, Hellberg G, Löwing K: Developmental profiles and auditory perception in 25 children attending special preschools for language-impaired children. Acta Paediatr 2002;91:1108-1115.
9 Tallal P: Auditory temporal perception, phonics, and reading disabilities in children. Brain Lang 1980;9:182-198.

$\checkmark 10$ Heath SM, Hogben JH, Clark CD: Auditory temporal processing in disabled readers with and without oral language delay. J Child Psychol Psychiatry 1999;40:637-647.

11 Tallal P: Fine-grained discrimination deficits in language-learning impaired children are specific neither to the auditory modality nor to speech perception. J Speech Hear Res 1990;33:616-621.

12 Miller CA, Kail R, Leonard LB, Tomblin JB: Speed of processing in children with specific language impairment. J Speech Lang Hear Res 2001;44:416-433.

13 Lahey M, Edwards J, Munson B: Is processing speed related to severity of language impairment? J Speech Lang Hear Res 2001;44: 1354-1361.

14 Bishop DVM, McArthur GM: Individual differences in auditory processing in specific language impairment: a follow-up study using event-related potentials and behavioural thresholds. Cortex 2005;41:327-341.

15 McArthur GM, Bishop DVM: Which people with specific language impairment have auditory processing deficits? Cognit Neuropsychol 2004;21:79-94.

16 McArthur GM, Bishop DM: Speech and non-speech processing in people with specific language impairment: a behavioural and electrophysiological study. Brain Lang 2005;94:260-273.

17 McArthur GM, Hogben JH: Auditory backward recognition masking in children with a specific language impairment and children with a reading disability. J Acoust Soc Am 2001;109:1092-1100.
18 Uwer R, Albrecht R, von Suchodoletz W: Automatic processing of tones and speech stimuli in children with specific language impairment. Dev Med Child Neurol 2002;44: 527-532.

19 Holopainen I, Korpilahti P, Juottonen K, Lang H, Sillanpää M: Attenuated auditory event-related potential (mismatch negativity) in children with developmental dysphasia. Neuropediatrics 1997;28:253-256.

20 Bishop DVM, Carlyon RP, Deeks JM, Bishop SJ: Auditory temporal processing impairment: neither necessary nor sufficient for causing language impairment in children. J Speech Lang Hear Res 1999;42:1295-1310.

21 Corriveau K, Pasquini E, Goswami U: Basic auditory processing skills and specific language impairment: a new look at an old hypothesis. J Speech Lang Hear Res 2007;50: 647-666.

22 Grimm H, Schöler H: Der Heidelberger Sprachentwicklungstest H-S-E-T, ed 2. Göttingen, Hogrefe, 1991.

23 Fischer B, Hartnegg K: On the development of low-level auditory discrimination and deficits in dyslexia. Dyslexia 2004;10:105118.

24 Brunner M, Seibert A, Dierks A, Körkel B: Heidelberger Lautdifferenzierungstest. Wertingen, Westra Elektroakustik, 1998.

25 McArthur GM, Bishop DVM: Auditory perceptual processing in people with reading and oral language impairment: current issues and recommendations. Dyslexia 2001;7: 150-170.

26 Hill PR, Hogben JH, Bishop DVM: Auditory frequency discrimination in children with specific language impairment. J Speech Lang Hear Res 2005;48:1136-1146. 